\title{
Duration of remission phase of 36 Korean patients with glossopharyngeal neuralgia
}

\author{
Myong-Soo Kang ${ }^{1}$, Do-Wan Kim , Sung-Min Kim ${ }^{1}$, Chan Kim², and Young-Ki Kim \\ ${ }^{1}$ Department of Anesthesiology and Pain Medicine, Ajou University Hospital, Suwon, ${ }^{2}$ Ki-Chan Pain Clinic, Seoul, ${ }^{3}$ Department of \\ Anesthesiology and Pain Medicine, Gangneung Asan Hospital, University of Ulsan College of Medicine, Gangneung, Korea
}

Background: Glossopharyngeal neuralgia has the characteristic of a long remission phase between the pain attack phases. Although the concept of remission is very important for the treatment of patients with glossopharyngeal neuralgia, due to the rarity of the disease, clear statistical studies on the remission phase for glossopharyngeal neuralgia are almost non-existent.

Methods: Previous chart reviews and phone interviews were conducted on a total of 38 patients. Among these study subjects, two patients were excluded because of their known secondary glossopharyngeal neuralgia from their brain tumors. Hence, the average duration of remission was investigated on 36 patients with idiopathic glossopharyngeal neuralgia.

Results: For the 27 patients who experienced their first remission, the average duration of the remission was 3.1 years. Among them, the average duration of the second remission of the 17 patients was 2.5 years, and for 4 patients who experienced a third remission, the average duration of the remission phase was 1.9 years.

Conclusions: The difference in the mean duration of the remission phase of the $1^{\text {st }}, 2^{\text {nd }}$, and $3^{\text {rd }}$ are not statistically significant, and the occurrence rate of the left or right side and of the gender, male or female, are also statistically insignificant. However, it is possible to infer that a patient might face a pain attack phase when his or her remission phase has lapsed for about three years. This prediction may be applied when developing treatment plans for patients with glossopharyngeal neuralgia. (Korean J Anesthesiol 2013; 64: 341-345)

Key Words: Glossopharyngeal neuralgia, Pain-free interval, Remission.

\footnotetext{
Received: December 19, 2011. Revised: 1st, April 4, 2012; 2nd, July 19, 2012; 3rd, September 16, 2012; 4th, September 29, 2012. Accepted: October 2, 2012. Corresponding author: Do-Wan Kim, M.D., Department of Anesthesiology and Pain Medicine, Ajou University Hospital, San 5, Woncheondong, Paldal-gu, Suwon 442-721, Korea. Tel: 82-31-219-5689, Fax: 82-31-219-5856, E-mail: anekinddo@naver.com (c) This is an open-access article distributed under the terms of the Creative Commons Attribution Non-Commercial License (http:// creativecommons.org/licenses/by-nc/3.0/), which permits unrestricted non-commercial use, distribution, and reproduction in any medium, provided the original work is properly cited.
} 


\section{Introduction}

Glossopharyngeal neuralgia was first described by Weisenburg in 1910 [1], and the prevalence in males and females was the same, with the most prevalent age group being between 40 to 60 years old, and the ratio between the prevalence in the left hand side to right hand side being about $3: 2$, where the prevalence in the left hand side was known to be greater than the right hand side [2]. Also, glossopharyngeal neuralgia is a very rare disease, where the prevalence rate in a year is about 0.7 persons $/ 100,000$ people [3-6], which is only $0.2-1.3 \%$ of the yearly prevalence rate of trigeminal neuralgia [7].

The typical characteristics and affected areas of glossopharyngeal neuralgia pain is shooting pain that radiates from the pharynx, tonsils, and the base of the tongue to the Eustachian tube and the inner ear or mandibular angle [8]. The symptoms of glossopharyngeal neuralgia are similar to trigeminal neuralgia, and like trigeminal neuralgia, the sharp and lancinating pain lasts for a few seconds to minutes and appears unilaterally. The paroxysmal pain is severe, which causes difficulties in all intentional activities of the patient, and can occur without any precipitating events, as well as with nonnoxious stimuli, such as talking, swallowing, inhalation of cold air, and light contact (facial washing, tooth brushing etc.) in the mouth or pharynx area.

Glossopharyngeal neuralgia may be caused by a structural lesion, secondary glossopharyngeal neuralgia, or occur without any structural lesions (idiopathic glossopharyngeal neuralgia). Some idiopathic glossopharyngeal neuralgia patients can have blood vessel compressions in the blood vessels around the glossopharyngeal nerve or the vagus nerve. In secondary glossopharyngeal neuralgia, the various tumors in the pharynx, tongue, cerebellopontine angle, and skull base are known to be the causes of shooting pain [1]. Also, secondary glossopharyngeal neuralgia can be suspected in cases where there are occurrences of neurological damage, such as numbness of the glossopharyngeal innervation, the absence of a remission period in between the pain attack phases, and pain radiating outside of the dermatome of the glossopharyngeal nerve [2,9].

There are intermission and remission phases between the each pain attack caused by glossopharyngeal neuralgia. The spans of intermissions vary; they can last for a few minutes to hours, or 1 to 2 days. They are so irregular that even a same patient can experience different intermission periods. The remission phase is part of the disease progression process that appears without specific treatment, and is normally known to last for a few weeks to years, with a finding reporting a maximum of 8 years [10]. This is called the pain-free interval. The remission phase is a characteristic of glossopharyngeal neuralgia. With the repetition of remission and pain attack after the initial onset, the remission phase gets shorter and the degree of pain during the pain attack phase becomes gradually severe [11]. However, for the investigation of the remission of glossopharyngeal neuralgia, most reports describe rough points based on the practitioner's clinical experiences due to the rare nature of this disease, with there being no statistically investigated studies.

In this study, the duration of remission was investigated in 38 patients who were diagnosed or treated for glossopharyngeal neuralgia and visited the outpatient clinic of this hospital once or more from January, 2010 till August, 2011 without follow-up loss.

\section{Materials and Methods}

From January, 2010 till August, 2011, amongst the 38 patients who were diagnosed with glossopharyngeal neuralgia, 2 patients with secondary glossopharyngeal neuralgia were excluded and only 36 patients with idiopathic glossopharyngeal neuralgia were included for this retrospective investigation of the age of onset, $1^{\text {st }}$ remission phase, $2^{\text {nd }}$ remission phase, and $3^{\text {rd }}$ remission phase through the chart reviews and interviews. Nine patients out of the 36 patients who continued to be under the remission phase since the $1^{\text {st }}$ pain attack phase and until August, 2011, and in this case, the duration of the remission phase cannot clearly be defined, so these cases were excluded from the data about the duration of the remission phase. However, 9 patients were included in the data for the age of onset, sex, and left/right sidedness.

The duration of the remission phase was investigated in a total of 27 patients. The pain attack phase was defined as when the patients feel pain to the degree that they cannot manage their daily activities, and it is generally the Visual Analogue Scale (VAS) of 8 to 10 . A remission phase was defined as the absence of pain between the attack phase or only the presence of bearable pain, where most of the patients described a degree of pain as being the Visual Analogue Scale of 7, which only the daily activities can barely be managed. With these references we could investigate up to the $5^{\text {th }}$ remission phase. Also, during the attack phase of glossopharyngeal neuralgia (VAS of $8-10$ ), all the patients were administered with carbamazepine (tegretol ${ }^{\circledR}$, Novartis, Seoul, Korea), of a minimum dose of $50 \mathrm{mg}$ tid to a maximum dose of $400 \mathrm{mg}$ tid according to the patient's degree of pain, and during admissions to the hospital the stellate ganglion block was carried out on a daily basis. After discharge from the hospital, the medication was tapered down for about 2 to 4 weeks according to the degree of pain reduction in all of the investigated patients, and the medication was discontinued when the VAS was 5 or below.

The remission phase was defined as a VAS being 7 or lower, but even patients who were in the remission phase with a VAS of 
about $6-7$, still complained about uncomfortable residual pain that interfered with the full management of their daily activities, therefore the medication was continuously administered until a VAS 5 or below was reached. Then, the medications were discontinued after interviews with the patients.

Seventeen out of the 36 patients who experienced a remission phase once or more, were administered carbamazepine until 2 weeks after their discharge from the hospital, 11 patients, 6 patients, and 2 patients were administered with carbamazepine until 3 weeks, 4 weeks, and 5 weeks, respectively after discharge from the hospital.

The exclusion of 9 patients, with their duration of remission phase being unknown as they continued to be in remission since the $1^{\text {st }}$ remission phase, 27 patients out of the total of 36 investigated patients experienced the $1^{\text {st }}$ remission phase, and among them, 17 patients experienced a $2^{\text {nd }}$ remission phase after going through the $1^{\text {st }}$ remission phase followed by the attack phase. Among these patients, 4 patients experienced a $3^{\text {rd }}$ remission phase. Only 1 patient experienced a $4^{\text {th }}$ and $5^{\text {th }}$ remission phase.

\section{Results}

The followings are the points in time when glossopharyngeal neuralgia first developed in the 36 patients: 6 patients in their 30s, 7 patients in their 40s, 10 patients in their 50s, 9 patients in their $60 \mathrm{~s}$, and 4 patients in their $70 \mathrm{~s}$, with the average age of onset being 54.1 years old. Twenty one patients developed glossopharyngeal neuralgia on the left hand side while the remaining 15 patients developed it on the right hand side. Nineteen patients were male while the remaining 17 patients were female (Table 1).

The average duration of the $1^{\text {st }}$ remission phase of the 17 patients who experienced a $1^{\text {st }}$ remission phase was 3.1 years, the average duration of the $2^{\text {nd }}$ remission phase of the 17 patients who experienced a $2^{\text {nd }}$ remission phase was 2.5 years, and the average duration of the $3^{\text {rd }}$ remission phase of the 4

Table 1. The Number of Patients According to Age, Sex, and Left/ Right Side Occurrences

\begin{tabular}{ccccc}
\hline Age & Male & Female & Left & Right \\
\hline $30 \mathrm{~s}$ & 3 & 3 & 1 & 5 \\
$40 \mathrm{~s}$ & 5 & 2 & 5 & 2 \\
$50 \mathrm{~s}$ & 5 & 5 & 6 & 4 \\
$60 \mathrm{~s}$ & 4 & 5 & 7 & 2 \\
$70 \mathrm{~s}$ & 2 & 2 & 2 & 2 \\
Total & 19 & 17 & 21 & 15 \\
\hline
\end{tabular}

GPN: Glossopharyngeal neuralgia. 30s: GPN onset of patients in their thirties, 40s: GPN onset of patients in their forties, 50s: GPN onset of patients in their fifties, 60s: GPN onset of patients in their sixties, and 70s: GPN onset of patients in their seventies. patients who experienced a $3^{\text {rd }}$ pain-free phase was 1.9 years (Table 2). Also, 9 patients who remained in the remissions phase after the $1^{\text {st }}$ pain attack phase, maintained the remission phase for an average of 26 months.

Looking at the pain-free periods in the different age groups, among the patients who developed the neuralgia in their $30 \mathrm{~s}$, the average duration of the $1^{\text {st }}$ remission phase was 2.6 years, the $2^{\text {nd }}$ remission phase was 2.4 years, and the $3^{\text {rd }}$ remission phase was 1.5 years. Among the patients who developed the neuralgia in their $40 \mathrm{~s}$, the average duration of the $1^{\text {st }}$ remission phase was 4.7 years, the $2^{\text {nd }}$ remission phase was 3.3 years, and the $3^{\text {rd }}$ remission phase was 2.0 years. Among the patients who the neuralgia developed in their 50 s, the average duration of the $1^{\text {st }}$ remission phase was 2.2 years, the $2^{\text {nd }}$ remission phase was 1.2 years, and the $3^{\text {rd }}$ remission phase was 2.0 years. Among the patients who developed the neuralgia in their $60 \mathrm{~s}$, the average duration of the $1^{\text {st }}$ remission phase was 3.5 years, the $2^{\text {nd }}$ remission phase was 2.3 years, and none of the patients experienced a $3^{\text {rd }}$ remission phase after developing glossopharyngeal neuralgia in their 60s. Among the patients who developed the neuralgia in their 70 s, the average duration of the $1^{\text {st }}$ remission phase was 1.3 years, the $2^{\text {nd }}$ remission phase was 5 years, and none of the patients experienced a $3^{\text {rd }}$ remission phase after developing glossopharyngeal neuralgia in their 70s. The average durations of each of the remission phases based on the age of onset are presented in Table 2. Patients who first developed glossopharyngeal neuralgia at the age of 58, and a duration of the $4^{\text {th }}$ and $5^{\text {th }}$ remission being 26 months and 34 months, respectively, were excluded from the data collection.

In the development of glossopharyngeal neuralgia on the left hand side, the average duration of the $1^{\text {st }}$ remission phase was 3.5 years, the $2^{\text {nd }}$ remission phase was 2.4 years, and the $3^{\text {rd }}$ remission was 2.0 years, while for the development on the right hand side, the average duration of the $1^{\text {st }}$ remission phase was 2.3 years, the $2^{\text {nd }}$ remission phase was 2.9 years, and

Table 2. Mean Remission Phase According to the Age Group

\begin{tabular}{cccc}
\hline Age & $\begin{array}{c}1^{\text {st }} \text { remission } \\
\text { phase }(\mathrm{yr})\end{array}$ & $\begin{array}{c}2^{\text {nd }} \text { remission } \\
\text { phase }(\mathrm{yr})\end{array}$ & $\begin{array}{c}3^{\text {rd }} \text { remission } \\
\text { phase }(\mathrm{yr})\end{array}$ \\
\hline $30 \mathrm{~s}$ & $(\mathrm{n}=5) 2.6 \pm 1.1$ & $(\mathrm{n}=3) 2.4 \pm 2.2$ & $(\mathrm{n}=1) 1.5 \pm 0$ \\
$40 \mathrm{~s}$ & $(\mathrm{n}=6) 4.7 \pm 2.7$ & $(\mathrm{n}=5) 3.3 \pm 1.4$ & $(\mathrm{n}=2) 2.0 \pm 0$ \\
$50 \mathrm{~s}$ & $(\mathrm{n}=7) 2.2 \pm 1.9$ & $(\mathrm{n}=3) 1.2 \pm 0.6$ & $(\mathrm{n}=1) 2.0 \pm 0$ \\
$60 \mathrm{~s}$ & $(\mathrm{n}=7) 3.5 \pm 1.6$ & $(\mathrm{n}=5) 2.3 \pm 1.3$ & \\
$70 \mathrm{~s}$ & $(\mathrm{n}=2) 1.3 \pm 0.5$ & $(\mathrm{n}=1) 5.0 \pm 0$ & \\
Total & $(\mathrm{n}=27) 3.1 \pm 2.2$ & $(\mathrm{n}=17) 2.5 \pm 1.7$ & $(\mathrm{n}=4) 1.9 \pm 0.2$
\end{tabular}

Values are the mean \pm SD. Difference in the $1^{\text {st }}, 2^{\text {nd }}$, and $3^{\text {rd }}$ mean remission phase according to the age group. The results are statistically insignificant. GPN: Glossopharyngeal neuralgia. 30s: GPN onset of patients in their thirties, 40s: GPN onset of patients in their forties, 50s: GPN onset of patients in their fifties, 60s: GPN onset of patients in their sixties, and 70s: GPN onset of patients in their seventies. 


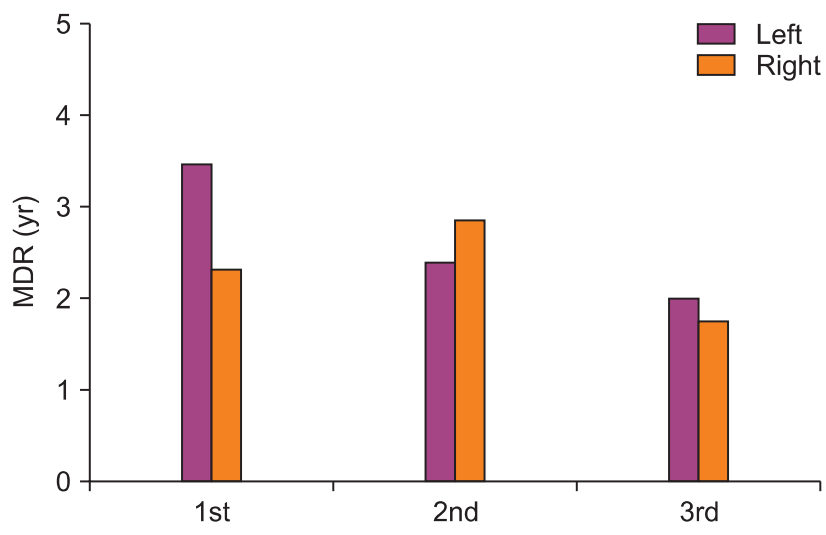

Fig. 1. Mean duration of remission according to the left or right side occurrence. MDR: Mean Duration of Remission Phase. The difference is statistically insignificant.

the $3^{\text {rd }}$ remission was 1.8 years (Fig. 1). Also, in male patients, the duration of the $1^{\text {st }}$ remission phase was 2.5 years, the $2^{\text {nd }}$ remission was 1.9 years, and the $3^{\text {rd }}$ remission was 2.0 years, while in female patients, the duration of the $1^{\text {st }}$ remission phase was 3.7 years, the $2^{\text {nd }}$ remission was 3.3 years, and the $3^{\text {rd }}$ remission was 1.8 years (Fig. 2).

There were no statistically significant differences between the average duration of the $1^{\text {st }}, 2^{\text {nd }}$, and $3^{\text {rd }}$ remission phase differences, while there were also no significant differences in the sex and age groups. SPSS (version 17.0, SPSS Inc, Chicago, USA) was used for the statistical analysis and the statistical significance was defined when $\mathrm{P}<0.05$.

\section{Discussion}

For the treatment of glossopharyngeal neuralgia, non-surgical treatment, such as medicinal treatment is first considered. Also, when the effects of medicinal treatment are not sufficient, surgical treatments, such as microvascular decompression can be considered. Generally, surgical treatment is more effective, but surgical treatment is related to a higher morbidity and prevalence rate than medicinal treatment $[8,12,13]$.

In glossopharyngeal neuralgia, patients who are not treated surgically, carbamazepine (tegretol $^{\circledR}$, Novartis, Seoul, Korea) is the first treatment of choice [14], where it has been reported that 2 to 3 divided doses of $200-800 \mathrm{mg}$ can lead to an $80 \%$ reduction of pain in patients [15]. However, with carbamazepine, symptoms of the central nervous system, such as narcolepsy, drowsiness, and ataxia, commonly occur [16], and long term use causes immune suppression, where reports of leukocytopenia and the reduction of the immunoglobulin antibody have been reported [17]. Also, carbamazepine has a high risk of tachyphylaxis, hence in the remission phase the medication is tapered down or ceased $[5,18]$. Therefore, the

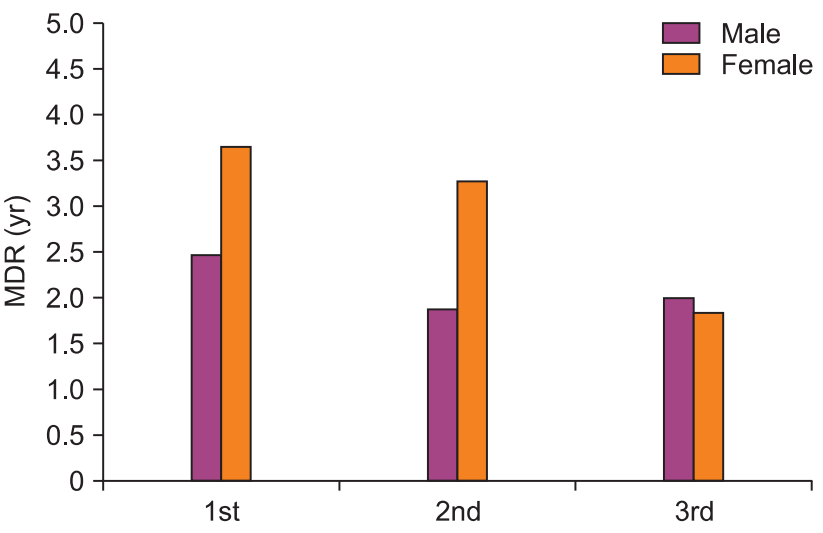

Fig. 2. Mean duration of remission phase according to the sex. MDR: Mean Duration of Remission Phase. The difference is statistically insignificant.

concept of the remission phase is a very critical factor that the physician who is treating the glossopharyngeal neuralgia patient needs to be aware of. However, as we have stated in the introduction, glossopharyngeal neuralgia is a very rare disease, so hardly any investigations are done with a large number of patients. In addition, there are claims that the actual prevalence of glossopharyngeal neuralgia is under reported due to the difficulty in diagnosis, difficulties in differential diagnosis with trigeminal neuralgia, and the lack of awareness of the disease itself [19]. In actuality, patients who have been diagnosed with glossopharyngeal neuralgia at this hospital have first been diagnosed with trigeminal neuralgia or atypical facial pain and toothache, therefore a detailed history taking and physical examination is needed for a more accurate diagnosis.

The significances of this study are; first, to find out the presence of the remission phase as a characteristic of glossopharyngeal neuralgia, and second, to prevent cases of increase in medication concentrations from tachyphylaxis and the lowered response to medication which may lead to the inability to use the medication, through the tapering of the medication with caution or the discontinuation of the medication during the remission phase. Also, with reference to the investigation of the average duration of the remission phase, the following theory can be used in real clinical practice after the onset and the pain attack phase, followed by the remission phase. If the remission phase has been greater than 3 years, then the relapse of pain may cautiously be explained to the patient.

In addition, the previously published papers reported that the prevalence between males and females are the same and the prevalence on the left hand side is higher than the right hand side, while the duration of the remission phase tends to be shorter with the repetition of the remission phase and the attack phase $[2,11]$. Certainly, the results of this study were aligned with previously published papers, but it was found not 
to be statistically significant. However, the number of patients who experienced $1^{\text {st }}, 2^{\text {nd }}$, and $3^{\text {rd }}$ remission phases were 27,17 , and 4 , respectively, and due to the small number of patients it is difficult to expect statistical significance.

In conclusion, there is the limitation in the difficulty in recruiting a large number of patients due to glossopharyngeal neuralgia, since it is a rare disease. A further study is warranted by recruiting a larger study population so that a more reliable study can be conducted in regards to the remission phase of glossopharyngeal neuralgia. In order to achieve this, the interests of the physicians to glossopharyngeal neuralgia and accurate diagnoses should be prioritized.

\section{References}

1. Weisenberg TH. Cerebellopontile tumor diagnosed for six years as tic douloureux: The symptoms of irritation of the 9th and 12th nerves. JAMA 1910; 54: 1600-4.

2. Bruyn GW. Glossopharyngeal neuralgia. Cephalalgia 1983;3:143-57

3. Katusic S, Williams DB, Beard CM, Bergstralh EJ, Kurland LT. Epidemiology and clinical features of idiopathic trigeminal neuralgia and glossopharyngeal neuralgia, Rochester, Minnesota, 1945-1984. Neuroepidemiology 1991; 10: 266-75.

4. Manzoni GC, Torelli P. Epidemiology of typical and atypical craniofacial neuralgias. Neurol Sci 2005; 26 Suppl 2: S65-7.

5. Rushton JG, Stevens JC, Miller RH. Glossopharyngeal (vagoglossopharyngeal) neuralgia: a study of 217 cases. Arch Neurol 1981; 38: 201-5.

6. Koopman JS, Dieleman JP, Huygen FJ, de Mos M, Martin CG, Sturkenboom MC. Incidence of facial pain in the general population. Pain 2009; 147: 122-7.

7. Evans RW, Torelli P, Manzoni GC.Glossopharyngeal neuralgia.
Headache 2006; 46: 1200-2.

8. Teixeira MJ, de Siqueira SR, Bor-Seng-Shu E. Glossopharyngeal neuralgia: neurosurgical treatment and differential diagnosis. Acta Neurochir (Wien) 2008; 150: 471-5.

9. Sobol SM, Wood BG, Conoyer JM. Glossopharyngeal neuralgiaasystole syndrome secondary to parapharyngeal space lesions. Otolaryngol Head Neck Surg 1982; 90: 16-9.

10. Peet MM. Glossopharyngeal neuralgia. Ann Surg 1935; 101: 256-68.

11. Barrow DL. Surgery of the cranial nerve of the posterior fossa. Philadelphia, Thieme Medical Pub. 1993, pp 245-50.

12. Edlich RF, Winters KL, Britt L, Long WB 3rd. Trigeminal neuralgia. J Long Term Eff Med Implants 2006; 16: 185-92.

13. Resnick DK, Jannetta PJ, Bissonnette D, Jho HD, Lanzino G. Microvascular decompression for glossopharyngeal neuralgia. Neurosurgery 1995; 36: 64-8.

14. Wiffen PJ, Derry S, Moore RA, McQuay HJ. Carbamazepine for acute and chronic pain in adult. Cochrane Database Syst Rev 2011; 19: CD005451.

15. Fromm GH, Terrence CF, Chattha AS. Baclofen in the treatment of trigeminal neuralgia: double-blind study and long-term follow-up. Ann Neurol 1984; 15: 240-4.

16. Rozen TD. Antiepileptic drugs in the management of cluster headache and trigeminal neuralgia. Headache 2001; 41 Suppl 1: S25-32.

17. Yamamoto T, Uchiyama T, Takahashi H, Himuro K, Kanai K, Kuwabara S. B cell aplasia and hypogammaglobulinemia after carbamazepine treatment. Intern Med 2010; 49: 707-8.

18. Cheshire WP. Trigeminal neuralgia: for one nerve a multitude of treatments. Expert Rev Neurother 2007; 7: 1565-79.

19. Gaul C, Hastreiter P, Duncker A, Naraghi R. Diagnosis and neurosurgical treatment of glossopharyngeal neuralgia: clinical findings and 3 -D visualization of neurovascular compression in 19 consecutive patients. J Headache Pain 2011; 12: 527-34. 